\title{
Cardiovascular Drugs and Therapy Celebrates its 25th Year of Publication with a New Section: Education in Cardiovascular Therapy
}

\author{
Willem J. Remme
}

Published online: 15 April 2011

(C) Springer Science+Business Media, LLC 2011

This year Cardiovascular Drugs and Therapy celebrates its 25th year of publication. In 1986 the journal, commonly known by its abbreviated title CDT, was founded by Henry Neufeld and Lionel Opie. Sadly, Henry Neufeld died suddenly before the first issue of CDT was published. Until 2001, when I took over as Editor-in-Chief, CDT was led and made to flourish by Lionel Opie, in the nineties together with the late Elliot Rapaport [1]. From the very beginning the journal was sponsored by the International Society of Cardiovascular Pharmacotherapy (ISCP) and later became its official journal.

Cardiovascular Drugs and Therapy is unique as it covers all pharmacological and pharmacotherapeutic aspects of the full range of cardiovascular and metabolic diseases including atherosclerosis, arrhythmias, CAD, hypertension, heart failure, hyperlipidemia, diabetes, peripheral vascular disease and others. It publishes original articles and reviews on basic pharmacology, clinical pharmacology, drugs and devices, clinical trials including trial design, and pharmaco-economics, -epidemiology and -genetics. Of much interest to our readership are the special sections and focused issues.

The journal has gradually increased its citation index, which currently stands at 2.515 . It receives much interest as shown by the large number of downloads $(>65.000$ in 2009) which can be found at the journal's website. Most importantly, we believe, is the fast handling of the manuscripts by the Editorial Board. Average time

W. J. Remme ( $\triangle)$

Sticares Cardiovascular Research Institute,

Oever 1-5, 3161GR,

Rhoon, The Netherlands

e-mail: w.j.remme@sticares.org between the date a manuscript is received and final decision is only 20.7 days. More than $80 \%$ of the articles are subsequently published on-line within 4 weeks. As this is exceptionally fast and as the impact of the journal is good, we should be satisfied with the status quo of the journal.

But we are not, at least not fully. What is missing in our view is a section in the journal providing timely information on currently important topics and structured to be of interest to basic scientists, clinical researchers and practicing clinicians alike. In fact, a section dealing with cardiovascular education from bench to bedside.

\section{Education in Cardiovascular Therapy}

In this issue the first set of articles in the new section on "Education in Cardiovascular Therapy" is published. I am very happy that Drs Bertram Pitt and Geoffrey S. Pitt have accepted to be the Editors in charge of this new section. Thanks to their input several topics of interest have been selected and expert authors approached, who have all accepted to contribute.

The topic in the current issue concerns the role of small non-coding RNAs in the failing heart. This series of manuscripts has been coordinated by Dr Douglas L. Mann from Washington University School of Medicine, St.Louis, USA, who also introduces the 3 related articles in this series [2-5]. The topic of small nc-RNAs is undoubtedly of major importance and, as Dr Mann indicates, likely to lead to novel diagnostic and therapeutic developments in heart failure. As such, a timely and from an educational point of view perfect introduction to this new series in Cardiovascular Drugs and Therapy. 


\section{References}

1. Opie L. In memoriam Elliot Rapaport. Cardiovasc Drugs Ther. 2011;25.

2. Mann DL. The emerging role of small non-coding RNAs in the failing heart: big hopes for small molecules. Cardiovasc Drugs Ther. 2011;25, this issue.
3. Holley CL, Topkara VK. An introduction to small non-coding RNAs: miRNA and snoRNA. Cardiovasc Drugs Ther. 2011;25, this issue.

4. Van de Vrie M, Heymans S, Schroen B. MicroRNA involvement in immune activation during heart failure. Cardiovasc Drugs Ther. $2011 ; 25$, this issue.

5. Topkara VK, Mann DL. Role of microRNAs in cardiac remodeling and heart failure. Cardiovasc Drugs Ther. 2011;25, this issue. 